\title{
数据挖掘驱动的 $\mathrm{BiFeO}_{3}-\mathrm{BaTiO}_{3}$ 铁电陶瓷元素 替代效应
}

姜英龙 ${ }^{1,2}$, 李军 ${ }^{1,2}$, 于剑 $2^{*}$

1. 东华大学物理系, 上海 201620 ;

2. 东华大学功能材料研究所, 上海 201620

*联系人, E-mail: jyu@dhu.edu.cn

2018-07-10 收稿, 2018-09-14 修回, 2018-09-17 接受, 2018-10-30 网络版发表 国家自然科学基金(61771122)资助

摘要传统的材料研究方式是耗时费力的试错法, 现代材料科学研究方式转向基于材料信息学(material informatics)理论的数据生产和利用——采用各种数据挖掘方法以发现材料数据下隐藏的构效关系和知识, 对新材 料进行预测和验证. 在总结过去对钙钛矿结构氧化物铁电材料数据挖掘实践结果的基础上，选择多种不同原(离) 子特征组合的元素对 $\mathrm{BiFeO}_{3}-\mathrm{BaTiO}_{3}(\mathrm{BF}-x \mathrm{BT})$ 铁电固溶体陶瓷进行替代实验, 重点探索三方-噟立方结构相界附近 组分的BF- $x \mathrm{BT}$ 基三元固溶体陶瓷的铁电相变和介电、压电响应性能. 实验发现替代元素的性质是 BF- $x \mathrm{BT}$ 基三元 固溶体陶瓷制备工艺条件和介电损耗、压电响应等电学性质的决定因素, $\mathrm{Bi}\left(\mathrm{Zn}_{1 / 2} \mathrm{Ti}_{1 / 2}\right) \mathrm{O}_{3}$ 是当前发现的对 $\mathrm{BF}-x \mathrm{BT}$ 基固溶体陶瓷进行改性、获得可工程实用的高性能高温压电陶瓷新材料的有效组元. 与现有商用偏铌酸铅和钛酸 铋系压电陶瓷材料相比, 它们可采用相同的固相反应电子陶瓷工艺制备、但具有更高压电响应等综合性能, 为研 制高灵敏高温压电传感器提供了新材料选项.

关键词钙钛矿结构氧化物, 数据挖掘, 压电陶瓷, 铁电相变, 压电响应

高灵敏、小型化、低成本、高稳定等压电传感器 的飞速发展势必要求压电陶瓷材料具有更新快、性能 好、增值高、污染少等优点 ${ }^{[1]}$. 现阶段商用压电陶瓷 依然是以 $\mathrm{Pb}(\mathrm{Zr}, \mathrm{Ti}) \mathrm{O}_{3}(\mathrm{PZT})$ 材料为主, 然而, PZT压电 陶瓷一方面在制备和使用过程中会对环境带来危害, 另一方面无法在高温环境下工作 ${ }^{[2]}$. 因此, 设计开发 高温、高压电响应、高温度稳定、低成本无铅/低铅 压电陶瓷材料成为材料工作者的重要任务. 虽然 $(\mathrm{K}, \mathrm{Na}) \mathrm{NbO}_{3}(\mathrm{KNN})^{[3]},\left(\mathrm{Bi}_{0.5}(\mathrm{Na}, \mathrm{K})_{0.5}\right) \mathrm{TiO}_{3}-\mathrm{BaTiO}_{3}{ }^{[4]}$ 和 $\mathrm{Ba}\left(\mathrm{Zr}_{0.2} \mathrm{Ti}_{0.8}\right) \mathrm{O}_{3}-\left(\mathrm{Ba}_{0.7} \mathrm{Ca}_{0.3}\right) \mathrm{TiO}_{3}{ }^{[5,6]}$ 等压电陶瓷近年来 获得广泛研究, 也展示出较好的压电响应, 然而这些 压电陶瓷的居里温度偏低、在居里温度以下还存在铁
电-(反)铁电相变, 导致这些陶瓷压电性能的温度稳 定性较差, 严重制约了它们的商业应用 ${ }^{[7,8]}$.

为了提高压电性能的温度稳定性, 有效策略是 设计开发具有较高居里温度且在居里温度以下无结构 相变的新型陶瓷材料体系 ${ }^{[8]} . \mathrm{BiFeO}_{3}-x \mathrm{BaTiO}_{3}(\mathrm{BF}-x \mathrm{BT}$, $x<0.4)$ 无铅压电陶瓷满足这样的要求, 在此组分范围 内存在一个三方-赝立方结构相界、铁电相变居里温 度 $T_{\mathrm{C}}>450^{\circ} \mathrm{C}$ 且 $T_{\mathrm{C}}$ 以下不存在结构相变, 因而有望得 到具有良好温度稳定性的高压电响应性能. 不幸的 是, 采用传统电子陶瓷工艺等方法制备的 BF- $x$ BT 压 电陶瓷表现为低直流电阻、高介电损耗, 使得 BF- $x$ BT 铁电陶瓷无法在较高电场下进行极化反转观测和压

引用格式: 姜英龙, 李军, 于剑. 数据挖掘驱动的 $\mathrm{BiFeO}_{3}-\mathrm{BaTiO}_{3}$ 铁电陶瓷元素替代效应. 科学通报, 2018, 63: 3229-3240 Jiang Y L, Li J, Yu J. Effects of substitution element on physical properties of $\mathrm{BiFeO}_{3}-\mathrm{BaTiO}_{3}$ ferroelectric ceramics driven by data-mining oxide perovskites (in Chinese). Chin Sci Bull, 2018, 63: 3229-3240, doi: 10.1360/N972018-00689 
电极化处理. 采用 $\mathrm{MnO}_{2}$ 掺杂和优化烧结制度, Leontsev和Eitel ${ }^{[9]}$ 在 $0.1 \%$ (重量百分比) $\mathrm{MnO}_{2}$ 掺杂的 $0.75 \mathrm{BF}-$ $0.25 \mathrm{BT}$ 压电陶瓷中, 观察到压电常数 $d_{33}=116 \mathrm{pC} / \mathrm{N}$, 介电常数 $\varepsilon_{r}=557$, 介电损耗 $\tan \delta=0.046$ (下文中未特 别指明的话介电损耗皆为 $1 \mathrm{kHz}$ 的数据), $T_{\mathrm{C}}=619^{\circ} \mathrm{C}$. 然而, 陶瓷材料的介电损耗离工程应用要求 $(\tan \delta \sim$ 0.02)尚有一段差距.

近年来, 我们在研究 $\mathrm{BiFeO}_{3}$ 的介电损耗问题时, 发现采用三元固溶体策略可有效提高铁酸铋钙钛矿 结构相的热力学稳定性, 能够稳定、重复获得高直流 电阻、低介电损耗的改性铁酸铋陶瓷材料 ${ }^{[10]}$. 在 BF- $x$ BT中加人亚稳态 $\mathrm{Bi}\left(\mathrm{Zn}_{1 / 2} \mathrm{Ti}_{1 / 2}\right) \mathrm{O}_{3}(\mathrm{BZT})$ 钙钛矿结 构氧化物形成三元固溶体即是一个很好的事例. 周 昌荣课题组 ${ }^{[11]}$ 报道了 $0.6 \%$ (重量百分比) $\mathrm{MnO}_{2}$ 掺杂 $0.71\left(\mathrm{BF}_{1-y} \mathrm{BZT}_{y}\right)-0.29 \mathrm{BT}(y=0.005 \sim 0.02)$ 陶瓷具有较大 的压电响应: $d_{33} \sim 160 \mathrm{pC} / \mathrm{N}$, 但他们未给出工程应用 重要的介电损耗数据. 赁敦敏课题组 ${ }^{[12]}$ 报道了 $1 \%$ (摩 尔百分比) $\mathrm{MnO}_{2}$ 掺杂的 $(0.70-y) \mathrm{BF}-0.30 \mathrm{BT}-y \mathrm{BZT}$ 压电 陶瓷, 在 $y=0.05$ 时具有极大压电响应性能: $d_{33}=139$ $\mathrm{pC} / \mathrm{N}, k_{p}=0.31, \varepsilon_{r}=650, \tan \delta=0.043, T_{\mathrm{C}}=529^{\circ} \mathrm{C}$. 本课 题组 ${ }^{[13 ~ 15]}$ 确定了 BF- $x$ BT- $y$ BZT三元固溶体的三方-赝 立方结构相界, 实验发现陶瓷的晶粒尺寸对相界位 置和压电响应有显著影响, 在 $0.27 \leqslant x \leqslant 0.31,0.01<$ $y<0.05$ 组分范围内, 粗晶陶瓷的 $d_{33} \geqslant 145 \mathrm{pC} / \mathrm{N}$. 优化 锰掺杂量和烧结工艺条件, $0.22 \%$ (重量百分比) $\mathrm{MnO}_{2}$ 掺杂0.696BF-0.29BT-0.014BZT粗晶陶瓷材料的压电 性能为 $d_{33}=150 \mathrm{pC} / \mathrm{N}, k_{p}=0.36, k_{t}=0.42, \varepsilon_{33}{ }^{\mathrm{T}} / \varepsilon_{0}=660$, $\tan \delta=0.033, T_{\mathrm{C}}=513^{\circ} \mathrm{C} ; 0.17 \%$ (重量百分比) $\mathrm{MnO}_{2}$ 掺杂 0.69BF-0.27BT-0.04BZT粗晶陶瓷的压电性能为 $d_{33}=$ $145 \mathrm{pC} / \mathrm{N}, k_{p}=0.33, k_{t}=0.41, \varepsilon_{33}{ }^{\mathrm{T}} / \varepsilon_{0}=570, \tan \delta=0.034$, $T_{\mathrm{C}}=510^{\circ} \mathrm{C}$. 它们具有优良的时间老化和热退极化老 化性能, 比如 $0.17 \%$ (重量百分比) $\mathrm{MnO}_{2}$ 掺杂 $0.69 \mathrm{BF}-$ 0.27BT-0.04BZT陶瓷样品, 经三年室温老化后, $d_{33}>$ $140 \mathrm{pC} / \mathrm{N}, \tan \delta \sim 0.033$, 在 $-40 \sim 100^{\circ} \mathrm{C}$ 温度范围内厚度 谐振频率和径向谐振频率的温度系数为 $-0.9 \%$, 厚度 机电耦合系数 $k_{t}$ 的温度系数为 $2.3 \%$. 这些工作表明, 钙钛矿结构BF-BT-BZT三元固溶体是研发高稳定性 无铅/低铅压电陶瓷的有效体系.

传统的新材料研究基于耗时费力的试错 (trialand-error)过程. 为了进一步理解和提高 BF- $x$ BT陶瓷 材料的压电性能, 本文将应用新近发展的材料信息 学理论方法, 在钙钛矿结构氧化物数据挖掘结果的
指导下, 重点研究三方-噟立方结构相界附近不同元 素替代对BF- $x$ BT陶瓷的铁电相变和压电响应性能的 影响.

\section{1 实验}

采用改进的固相反应电子陶瓷工艺 ${ }^{[13 ~ 15]}$ 制备 $\mathrm{Mn}$ 掺杂BF- $x \mathrm{BT}-y \mathrm{ABO}_{3}$ 系列三元固溶体陶瓷样品 $\left(\mathrm{ABO}_{3}=\right.$ $\mathrm{Bi}\left(\mathrm{Zn}_{1 / 2} \mathrm{Ti}_{1 / 2}\right) \mathrm{O}_{3}, \quad \mathrm{BiScO}_{3}, \quad \mathrm{BiAlO}, \quad \mathrm{Ba}\left(\mathrm{Bi}_{1 / 2} \mathrm{Nb}_{1 / 2}\right) \mathrm{O}_{3}$, $\mathrm{Ba}\left(\mathrm{Zn}_{1 / 3} \mathrm{Nb}_{2 / 3}\right) \mathrm{O}_{3}, \mathrm{BaZrO}_{3}$ 等 $)$, 所用原料及其纯度为: $\mathrm{Bi}_{2} \mathrm{O}_{3}\left(99 \%\right.$ Alfa Aesar), $\mathrm{Fe}_{2} \mathrm{O}_{3}(99.945 \%$ Alfa Aesar), $\mathrm{BaCO}_{3}\left(99.95 \%\right.$ Alfa Aesar), $\mathrm{TiO}_{2}(99.9 \%$ Alfa Aesar), $\mathrm{ZnO}(99.5 \%$ Acros $), \mathrm{Nb}_{2} \mathrm{O}_{5}\left(99.9 \%\right.$ Alfa Aesar), $\mathrm{ZrO}_{2}$ (99\% Alfa Aesar), $\mathrm{Sc}_{2} \mathrm{O}_{3}\left(99.9 \%\right.$ Aladdin), $\mathrm{Al}_{2} \mathrm{O}_{3}(\mathrm{AR}$, SCRC) 和 $\mathrm{MnO}_{2}(99.9 \%$ Alfa Aesar). 根据设计的组分 按化学计量比进行计算和称量, 在玛瑙研钵里用无 水乙醇做助磨剂手工研磨 $2 \mathrm{~h}$. 磨好的粉料在 $700^{\circ} \mathrm{C}$ 下 预烧保温 $5 \mathrm{~h}$, 预烧后的粉料用同样的方法手工研磨 $2 \mathrm{~h}$, 在 $780^{\circ} \mathrm{C}$ 下进行第2次预烧保温 5 h. 2 次预烧后, 粉料再次进行手工研磨, 并加聚乙烯醇(PVA)黏结剂 造粒, 随后在 $200 \mathrm{MPa}$ 下将粉料压成圆片样品. $600^{\circ} \mathrm{C}$ 保温 $2 \mathrm{~h}$ 排黏; 对不同组分样品, 在 $900 \sim 930^{\circ} \mathrm{C}$ 范围选 择合适的温度进行若干小时烧结, 烧结时样品放置 在密闭的氧化铝坩埚内, 烧结后样品随炉冷却.

使用X射线衍射(XRD)技术测量样品的晶体结 构. 样品的断面形貌采用扫描电子显微镜(SEM)进行 表征. 进行电性能测试前, 将烧结后的样品进行表面 抛光处理, 在样品表面涂覆银浆, 涂好银浆的样品在 $650^{\circ} \mathrm{C}$ 下保温 $10 \mathrm{~min}$ 后随炉冷却. 用 $\mathrm{LCR}$ 数字电桥对 样品进行室温介电常数和介电损耗频谱测量, 进行 高温介电常数和介电损耗温谱测量. 样品在 $120^{\circ} \mathrm{C}$ 的 硅油中极化, 施加 $8 \mathrm{kV} / \mathrm{mm}$ 的电场并保压 $15 \mathrm{~min}$, 将 已极化好的样品放置 $24 \mathrm{~h}$, 采用中国科学院声学研究 所的准静态压电系数 $d_{33}$ 测量仪 $(\mathrm{ZJ}-6 \mathrm{~A})$ 测试样品的压 电常数.

\section{2 结果讨论与分析}

近年来, 数据挖掘方法和信息理论在材料科学 领域拓展其应用, 一门被称之为材料信息学(material informatics) 的理论方法已开始应用于发现材料数据 下隐藏的构效关系, 并对新材料进行预测 ${ }^{[16]}$. 在数 据科学范式下, 借助数据挖掘的算法模型和相关领 域专业知识(domain knowledge), 通过发现数据间的 因果(causal)关系或相干(correlation)关系, 材料工作 
者开始尝试建立 “按需设计”的新材料预测理论和方 法. 不同于既费时又费力的试错式实验和数值模拟 方法, 材料信息学方法基于过去已存在的可靠数据 或实验中产生的新数据, 对每一材料对象选择合适 的数字化描述符, 通过机器学习等数据挖掘方法建 立目标性质与描述符之间的关联关系, 采用内插/外 推等方法进行新材料对象及其性能预测 ${ }^{[17]}$. 在此过 程中, 有关材料特征 (fingerprint) 的数字化描述符的 选择不是那么直接和显而易见的, 需要借助不同材 料领域的专业知识和经验, 并且与所关注的性质或 现象紧密相关; 同时, 机器学习等统计算法模型经常 遇到过拟合 (overfitting) 问题, 即算法模型虽然可以 准确拟合手头的材料数据、但无法合理解释其关联关 系、由此导致对新材料的一些不正确预测 ${ }^{[18]}$. 因此, 有效集成数据算法模型和基于材料理论的物理模型 是材料信息学研究的一大挑战, 我们不能只是应用 机器学习进行数据的统计拟合、而应该认识到: 即使 是同一性质参数也可能有多种物理化学机制、要先对 材料进行分门别类再进行描述符选择和构效关系的 数学拟合, 经循环迭代获得物理的算法预测模 型 ${ }^{[19,20]}$.

选择好的描述符是理解和预测材料性质的基石 和关键. 凝聚态物理理论告诉我们, 材料的宏观物理 行为是原子系统集体的呈展 (emergence)现象, 很多 时候无法直接用原子的特征量一原子序数、元素周 期号、离子半径、电负性等来描述原子堆积系统的结 构及其相关性质 ${ }^{[21]}$. 铁电物理领域知识 (domain knowledge)表明, 原胞约合质量、结构容忍因子、 $\mathrm{A} / \mathrm{B}$ 位离子半径比等是钙钛矿结构氧化物 $\left(\mathrm{ABO}_{3}\right)$ 很好的 数字化描述符, 它们可以直接从原(离)子的特征参数 计算得到, 与所研究的材料化学式一一对应 ${ }^{[22]}$. 晶格 动力学相变理论表明, 当涨落(包括热涨落和量子零 点涨落)与铁性序参量的大小相等时, 钻钛矿结构氧 化物将发生结构相变: 结构相变温度可以用涨落的描 述符量化描述、相变性质由铁性序参量的性质决 定 $^{[23]}$. 通过对钙钛矿结构氧化物进行数据挖掘, 本课 题组 ${ }^{[24,25]}$ 发现: (1) 铁电相变的居里温度 $T_{\mathrm{C}}$ 与原胞约 合质量 $(\mu)$ 之间满足量化关系: $T_{\mathrm{C}}(\mu)=a+b \mu+c \mu^{2}$, 系数 $(a, b$ 和 $c$ ) 对特定的元素组是常数 (即可以用该系 数组对元素性质进行分类); (2) 结构容忍因子 $(t)$ 是预 测铁弹相变的好的描述符: $t=1$ 时晶体为立方相, $t>1$ 时发生立方-四方铁弹结构相变, $t<1$ 时发生立方-三
方(正交、单斜)铁弹相变. 结构容忍因子虽然与铁电 相变没有直接的关联，但却是预测固溶体材料准同 型结构相界位置的较好描述符 ${ }^{[26,27]}$; (3) 只有当铁电 活性离子的体积小于配位氧离子笼的间隙(间隙体积 的大小同时包括动能项和势能项的贡献)时, 铁电活 性离子将离开配位氧离子笼的对称中心位置, 从而 导致对称中心破缺和铁电序参量的产生 ${ }^{[25]}$. 铁电活 性离子偏离对称中心的距离是预测铁电结构相变的 较好描述符, 它不仅提供了铁电性产生的原子尺度 的物理起源、还可以直接用于描述铁电序参量的强 度 $^{[28 ~ 30]}$.

基于上述数据挖掘结果, 对钙钛矿结构 BF- $x \mathrm{BT}$ 固溶体陶瓷进行了不同的元素替代以调控其铁电相 变居里温度和相关物理性质, 元素选择基于不同的 原子质量(原胞约合质量、轻重)和 $\mathrm{A} / \mathrm{B}$ 位离子半径比 (大小)组合, 具体详情见表 $\mathrm{S} 1$. 前期实验已知 $\mathrm{BiFeO}_{3}$ 和 $\mathrm{BaTiO}_{3}$ 晶体分别为钙钛矿结构三方和四方相, 它 们能够形成连续二元固溶体，在富 BF组分区域存在 一个三方-赝立方结构相界 ${ }^{[9,31 ~ 34]}$. 在钻钛矿结构PZT 和BF-BZT-PT等压电陶瓷的实验研究中, 发现晶粒 尺寸也是调控结构相界位置和压电响应性能的一个 重要手段 ${ }^{[35,36]}$. 结合陶瓷晶粒尺寸等微结构观测, $x=$ 0.275 曾被确定为 BF- $x$ BT 细晶 $(\sim 1 \mu \mathrm{m})$ 陶瓷的结构相 界 ${ }^{[32]} 、 x=0.33$ 为粗晶 $(\sim 5 \mu \mathrm{m})$ 陶瓷的结构相界 ${ }^{[33,34]}$.

选择0.73BF-0.27BT进行 $\mathrm{Al}, \mathrm{Sc}$ 和 $\mathrm{Zn}_{1 / 2} \mathrm{Ti}_{1 / 2}$ 替代 $\mathrm{Fe}$ 实验, 选择0.69BF-0.31BT进行 $\mathrm{Bi}_{1 / 2} \mathrm{Nb}_{1 / 2}, \mathrm{Zn}_{1 / 3} \mathrm{Nb}_{2 / 3}$, $\mathrm{Zr}$ 等替代 $\mathrm{Ti}$ 实验, 同时也进行了高 $\mathrm{BF}$ 含量的 $\mathrm{BF}-x \mathrm{BT}$ $0.04 \mathrm{BZT}$ 实验. 采用改进的固相反应电子陶瓷工艺, 制备了表 $\mathrm{S}$ 1所列不同组分 $\mathrm{MnO}_{2}$ 掺杂 $\mathrm{BF}-x \mathrm{BT}$ 基钙钛 矿结构固溶体陶瓷. 采用 X射线衍射 $(X R D)$ 测试了陶 瓷粉末的晶体结构, 典型结果如图1所示. 在XRD测 试时, 衍射角 $2 \theta$ 在 $39^{\circ}$ 附近的衍射峰可以作为区分三 方与赝立方相的探针 ${ }^{[13 ~ 15]: ~} 1$ 个衍射峰对应赝立方相, 对应 $(111)_{\mathrm{pc}}$ 晶面; 而 2 个衍射峰对应三方相, 对应 $(006)_{\mathrm{R}}$ 与 $(202)_{\mathrm{R}}$ 晶面. 在Origin图形软件中进行峰形 拟合, 如图1所示, BF65-BT32-BZT3样品可用一个对 称的Voigt形峰进行拟合, 而BF74-BT22-BZT4, BF69-BT27-BZT4, BF69-BT27-BiScO 3 , BF69-BT27$\mathrm{BiAlO}_{3}$ 和BF69-BT27-BZT4+0.003ST+0.023PT等样品 是 2 个对称Voigt形峰叠加的结果. 结合BF-BT二元固 溶体相图和XRD测试结果, BF65-BT32-BZT3陶瓷是 钙钛矿结构赝立方相, 图1显示的其他样品是三方相, 


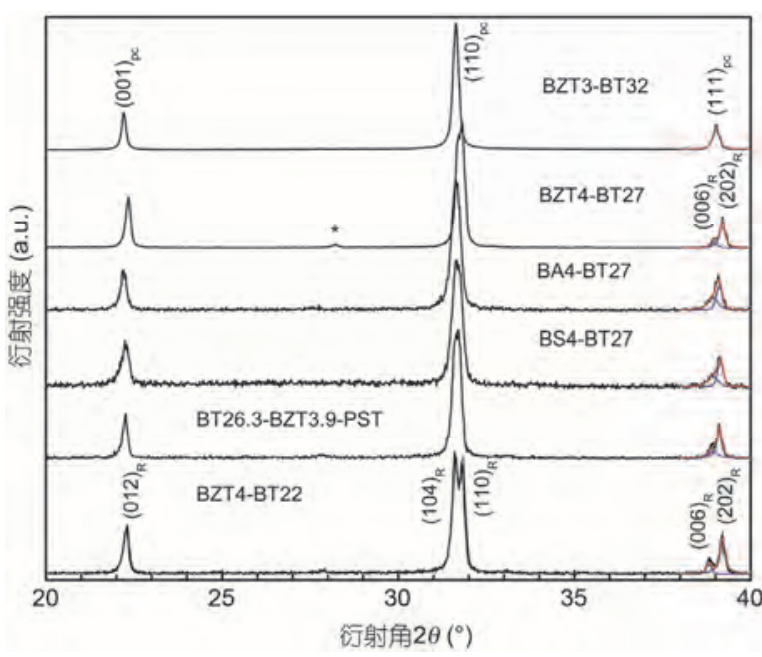

图 1 不同元素替代BF- $x$ BT陶瓷样品室温粉末 $X$ 射线衍射图谱. BZT3-BT32:BF65-BT32-BZT3, BZT4-BT27:BF69-BT27-BZT4, BS4BT27:BF69-BT27-BiScO 3 , BA4-BT27:BF69-BT27-BiAlO 3 , BT26.3BZT3.9-PST:BF69-BT27-BZT4+0.003ST+0.023PT, BZT4-BT22:BF74BT22-BZT4. 其中, 用*标记的衍射峰来自类 $\gamma-\mathrm{Bi}_{2} \mathrm{O}_{3}$ 结构杂相. $\mathrm{R}$ 和pc 下角标分别代表三方和赝立方相. 蓝色线代表Voigt形拟合峰, 红色 线代表拟合结果

Figure 1 Powder X-ray diffraction patterns recorded at room temperature for various BF- $x \mathrm{BT}$-based ternary solid solution ceramics: BF65BT32-BZT3 (labelled as BZT3-BT32), BF69-BT27-BZT4 (BZT4BT27), BF69-BT27-BiScO 3 (BS4-BT27), BF69-BT27-BiAlO ${ }_{3}$ (BA4BT27), BF69-BT27-BZT4+0.003ST+0.023PT (BT26.3-BZT3.9-PST) and BF74-BT22-BZT4 (BZT4-BT22). The peak marked by star * come from impurity phase in $\gamma-\mathrm{Bi}_{2} \mathrm{O}_{3}$-isostructure. $\mathrm{R}$ and pc subscript for rhombohedral and pseudocubic phase, respectively. Blue and red lines are fitting results of Voight-shaped peaks and their sum in Origin software, respectively

更多结构指认结果见表S1. (006)与(202)衍射峰䢃裂 程度表明, BF69-BT27-BZT4, BF69-BT27-- $\mathrm{ABO}_{3}$ 和 BF69-BT27-BZT4+0.003ST+0.023PT样品靠近三方噟立方结构相界, 而BF74-BT22-BZT4的组分远离相 界. 需要指出的是, 对于BF69-BT27-BZT4样品, 星 号标示的衍射峰来源于类 $\gamma-\mathrm{Bi}_{2} \mathrm{O}_{3}$ 结构第二相. 这是 因为BZT是钙钛矿结构亚稳相, 它与BF-BT只能形成 有限固溶体; 同时 $\mathrm{Bi}_{2} \mathrm{O}_{3}$ 是很好的熔剂, 接近或超过 固溶限后形成的非钙钛矿结构第二相具有类 $\gamma-\mathrm{Bi}_{2} \mathrm{O}_{3}$ 的晶体结构, 从热力学角度讲它是比较稳定的 ${ }^{[13]}$.

铁酸铋 (BF) 钙钛矿结构氧化物是磁电、压电多重 铁性功能材料探索的一个典型研究对象(playground). 从电学性能的直接实验测量和工程应用需求出发, 制备高阻、低介电损耗 $(\tan \delta)$ 陶瓷样品是进行性能评 估、获得可靠实验数据的前提. 由于 $\mathrm{BiFeO}_{3}$ 钙钛矿结 构相的热力学稳定性较低、 $\mathrm{Bi}$ 元素的挥发性等原因, 传统电子陶瓷工艺制备的 BF- $x$ BT等铁酸铋系陶瓷的
介电损耗都比较大，比如先前报道BF72.5-BT27.5和 $\mathrm{BF} 70-\mathrm{BT} 30$ 陶瓷的 $\tan \delta$ 分别为 $0.078^{[32]}$ 与 $0.088^{[34]}$; 通 过掺杂 $0.1 \%$ (重量百分比) $\mathrm{MnO}_{2}$ 和优化烧结条件, BF75-BT25 和 BF67-BT33 压电陶瓷的 $\tan \delta$ 降低到 0.046 和 $0.068^{[9]}$. 通过对影响铁酸铋陶瓷介电损耗的 因素进行数据挖掘和相应的实验验证, 本课题 组 ${ }^{[10,24]}$ 发现铁酸铋钻钛矿结构相的热力学稳定性较 低是导致低电阻、高介电损耗的主导因素，相比于形 成二元固溶体, $\mathrm{BiFeO}_{3}$ 钙钛矿结构相的热力学稳定性 可通过形成三元固溶体获得极大提高、 $\mathrm{Bi}$ 的挥发也得 到抑制. 按化学计量比投料、采用改进的固相反应电 子陶瓷工艺 $890^{\circ} \mathrm{C}$ 烧结制备的 $\mathrm{Bi}_{0.98} \mathrm{La}_{0.02} \mathrm{Fe}_{0.99} \mathrm{Ti}_{0.01} \mathrm{O}_{3}$ 陶瓷的 $\tan \delta$ 小于 $0.02,780{ }^{\circ} \mathrm{C}$ 以上温度烧结制备的 BF- $x$ PT $-y$ BZT 陶瓷在较宽的组分范围内 $\tan \delta \sim$ $0.02^{[37 ~ 39]}$. 这是本文开展BF- $x$ BT 系铁电压电陶瓷研 究的出发点和基础之一.

对不同元素替代的BF- $x$ BT陶瓷样品进行了室温 介电频谱测量, 图2给出了部分典型结果, 更多结果 总结见表 $\mathrm{S} 1$. 对比图 2 和表 $\mathrm{S} 1$ 可见，不同的元素替代 对获得低介电损耗的BF- $x$ BT 陶瓷样品有不同的效 果、需要不同的 $\mathrm{Mn}$ 掺杂量. 这是因为, 高温烧结时由 于 $\mathrm{Bi}$ 元素的挥发, 在钙钛矿结构晶格中会留下 $\mathrm{A}$ 位空 位和相应浓度的O空位，导致较大的介电损耗; 而不 同的元素替代对 $\mathrm{Bi}$ 元素挥发的抑制能力不同. 掺杂 一定量的 $\mathrm{MnO}_{2}, \mathrm{Mn}$ 离子会占据B位，高温时价态会

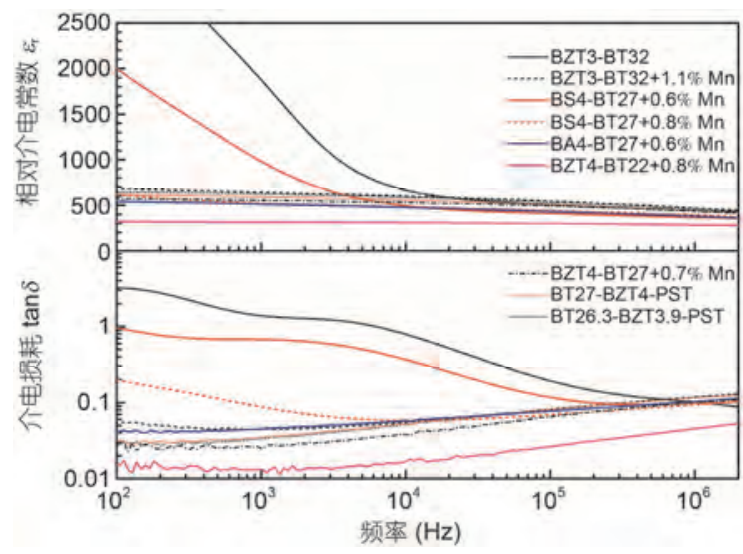

图 2 不同元素替代BF- $x$ BT系陶瓷样品的室温介电频谱图: BF69BT27-BZT4+0.003ST+0.003PT (BT27-BZT4-PST), 其余样品简写与 图 1 相同

Figure 2 Frequency-dependent dielectric constant and loss spectra recorded at room temperature for various BF- $x$ BT-based ternary solid solution ceramics: BF69-BT27-BZT4+0.003ST+0.003PT (BT27-BZT4PST), other samples same as those in Figure 1 
升高, 从而大幅降低 $\mathrm{O}$ 空位浓度. 虽然掺杂少量 $\mathrm{Mn}$ 会增加 $\mathrm{A}$ 位空位浓度, 但由于 $\mathrm{O}$ 空位浓度的降低, 可 以有效降低BF-BT基压电陶瓷的介电损耗 ${ }^{[13 \sim 15]}$. 与 BF69-BT27-BZT4相比, BF65-BT32-BZT3的BZT含量 降低、BT含量增加, $1.1 \%$ (摩尔百分比)的 Mn掺杂量才 使得 $\tan \delta$ 降低到 0.044; BF69-BT27- $\mathrm{BiScO}_{3}$ 掺杂 $0.8 \%$ (摩尔百分比) $\mathrm{Mn}$ 介电损耗才降低至 0.087 ; 而 $0.6 \%$ (摩 尔百分比) $\mathrm{Mn}$ 掺杂 $\mathrm{BF} 69-\mathrm{BT} 27-\mathrm{BiAlO}_{3}$ 陶瓷的 $\tan \delta$ 降 低至 0.045 . 对于 $0.6 \%$ (摩尔百分比) Mn掺杂 BF69BT27- $\mathrm{BaBO}_{3}\left(\mathrm{~B}=\mathrm{Bi}_{1 / 2} \mathrm{Nb}_{1 / 2}, \mathrm{Al}_{1 / 2} \mathrm{Nb}_{1 / 2}, \mathrm{Zn}_{1 / 3} \mathrm{Nb}_{2 / 3}, \mathrm{Zr}\right.$ 和 $\mathrm{Sn})$ 陶瓷, 如表 $\mathrm{S}$ 所示, 本实验获得的最低介电损耗为 $\tan \delta=0.056$. 与 $\mathrm{B}$ 位元素替代不同, 少量的 $\mathrm{Pb}, \mathrm{Sr}$ 元素 进行替代, 如BF69-BT27-BZT4+0.003ST+0.003PT和 BF69-BT27-BZT4+0.003ST+0.023PT陶瓷, 在本文实 验条件范围内即与 $0.17 \%$ (重量百分比) $\mathrm{MnO}_{2}$ 掺杂 BF69-BT27-BZT4 的损耗在同一水平 $(\tan \delta \sim 0.033)$. 增加BF的含量, 也可降低介电损耗, 如BF74-BT22BZT4陶瓷的 $\tan \delta=0.010$.

对比不同元素的替代效果可以发现：通过调控 $\mathrm{Mn}$ 掺杂量和烧结条件, BF- $x$ BT- $y$ BZT三元固溶体很 容易获得低介电损耗的陶瓷材料, 比如: 赁敦敏课题 组 ${ }^{[12]}$ 制备的 $1 \%$ (摩尔百分比) $\mathrm{MnO}_{2}$ 掺杂 $(0.70-x) \mathrm{BF}-$ $x \mathrm{BZT}-0.30 \mathrm{BT}(x=0.0 \sim 0.15)$ 压电陶瓷 $\tan \delta=0.043$; 本课 题组 ${ }^{[13 ~ 15]}$ 前期制备的 $0.22 \%$ (重量百分比) $\mathrm{MnO}_{2}$ 掺杂 0.696BF-0.29BT-0.014BZT和0.17\%(重量百分比) $\mathrm{MnO}_{2}$ 掺杂0.69BF-0.27BT-0.04BZT压电陶瓷的介电损耗分 别为 0.033 和 0.034. 近来, 通过调控 $\mathrm{Mn}$ 含量 (图 2), $0.7 \%$ (摩尔百分比)Mn掺杂BF69-BT27-BZT4和 $0.8 \%$

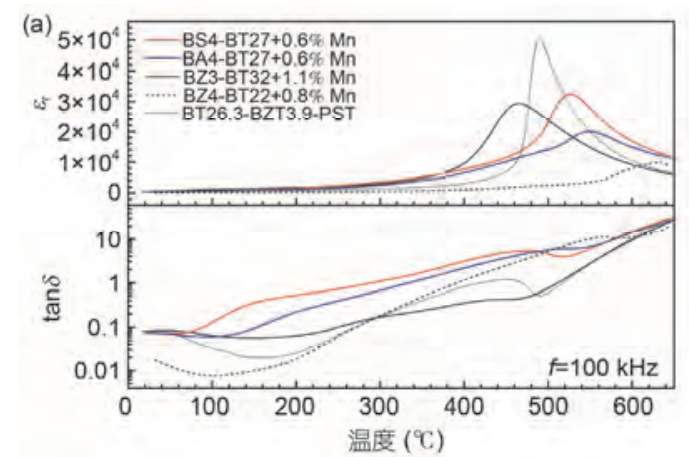

$\mathrm{Mn}$ (摩尔百分比)掺杂BF74-BT22-BZT4陶瓷样品, 本 课题组获得了 $\tan \delta \sim 0.02$ 及其以下工程应用需求水平 的BF-BT-BZT无铅压电陶瓷. 广泛的实验和数据挖 掘表明, BZT是有效提高 $\mathrm{BF}-x \mathrm{BT}$ 系钙钛矿结构相热 力学稳定性的第三组元, 不同的BZT浓度对 Bi的挥 发抑制能力不同: BZT含量越高、对Bi挥发的抑制能 力越高, 直至完全抑制, 此时没有 $\mathrm{Mn}$ 掺杂辅助手段 也能够获得 $\tan \delta \sim 0.02$ 及其以下的介电损耗性质 ${ }^{[13]}$.

选取那些室温介电损耗较低的陶瓷样品进行高 温介电性质测量, 部分典型测试结果见图3(a). 如图 所示, 介电常数的峰值温度对应铁电-顺电相变居里 温度 $T_{\mathrm{C}}$; 对不同元素替代BF- $x$ BT 陶瓷测量得到的相 变温度 $T_{\mathrm{C}}$ 总结于表 $\mathrm{S} 1$. 程晋荣课题组 ${ }^{[31]}$ 测试了不同 含量 BF- $x$ BT陶瓷铁电相变居里温度：例如 $1.0 \%$ (摩尔 百分比) $\mathrm{Mn}$ 掺杂 $0.69 \mathrm{BF}-0.31 \mathrm{BT}$ 铁电陶瓷的 $T_{\mathrm{C}}=479^{\circ} \mathrm{C}$, $0.73 \mathrm{BF}-0.27 \mathrm{BT}$ 陶瓷的 $T_{\mathrm{C}}=532^{\circ} \mathrm{C}$; 对 BF- $x \mathrm{BT}$ 二元固溶 体采用原胞约合质量为描述符重新作图, 如图3(b)实 线所示, 拟合发现它们很好地满足 $T_{\mathrm{C}}(\mu)=a+b \mu+c \mu^{2}$ 量化关系. 然而, 广泛地对 BF- $x$ BT 铁电陶瓷 $T_{\mathrm{C}}$ 的测 试结果表明, 铁电相变居里温度受 $\mathrm{Mn}$ 掺杂量、烧结 条件(不同的介电损耗水平、不同的陶瓷微结构晶粒 尺寸大小)等多种因素影响 ${ }^{[9,31 \sim 34,40]}$; 与程晋荣课题 组结果相比, 存在相当程度的发散(图3(b)), 未来需 要仔细记录实验的相关参数以量化不同因素对居里 温度的贡献. 从图3(a)我们可以确定 $0.6 \%$ (摩尔百分 比) Mn掺杂BF65-BT32-BZT3铁电陶瓷的 $T_{\mathrm{C}}=463^{\circ} \mathrm{C}$, 低于 $0.17 \%$ (重量百分比) $\mathrm{MnO}_{2}$ 掺杂BF69-BT27-BZT4 陶瓷的居里温度 $\left(T_{\mathrm{C}}=510^{\circ} \mathrm{C}\right)$, 而 $0.8 \%$ (摩尔百分比)

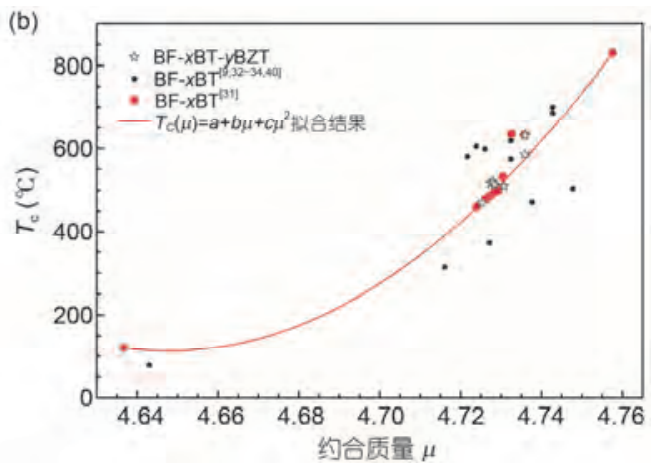

图 3 (网络版彩色) 不同元素替代BF- $x$ BT系陶瓷铁电相变测试. (a) 介电温谱图, 测试频率为 $100 \mathrm{kHz}$; (b) 铁电相变居里温度 $\left(T_{\mathrm{C}}\right.$ )与钲钛矿结构 氧化物原胞约合质量 $(\mu)$ 的关系. BF- $x$ BT- $y$ BZT铁电陶瓷的部分结果见文献[13 15]

Figure 3 (Color online) Measurements of ferroelectric structural phase transition of various BF- $x$ BT-based ternary solid solution ceramics. (a) Temperature-dependent dielectric constant and loss spectra recorded at $100 \mathrm{kHz}$ frequency. (b) Plotting of ferroelectric Curie temperatures ( $T_{\mathrm{C}}$ ) with respect to reduced mass of perovskite unit cell $(\mu)$. Some results for BF- $x$ BT- $y$ BZT ferroelectric ceramics cited from Refs. [13-15] 
$\mathrm{Mn}$ 掺杂的BF74-BT22-BZT4陶瓷的 $T_{\mathrm{C}}=630^{\circ} \mathrm{C}$. 目前 的实验结果表明, 当BZT含量 $y<0.05$ 时, BF-BT-BZT 三元固溶体居里温度对 $\mathrm{BT}$ 含量的变化趋势与 $\mathrm{BF}-x \mathrm{BT}$ 二元固溶体陶瓷的一致: 即降低BT含量、增加 $\mathrm{BF}$ 含 量居里温度升高(图3(b)). 用 $\mathrm{Al}, \mathrm{Sc}$ 替代等摩尔量的 $\mathrm{Fe}$, 实验测得 $0.6 \%$ (摩尔百分比) Mn掺杂BF69-BT27$\mathrm{BiAlO}_{3}$ 铁电陶瓷的 $T_{\mathrm{C}}=549^{\circ} \mathrm{C}, 0.6 \%$ (摩尔百分比) $\mathrm{Mn}$ 掺杂 $\mathrm{BF} 69-\mathrm{BT} 27-\mathrm{BiScO}_{3}$ 陶瓷的 $T_{\mathrm{C}}=525^{\circ} \mathrm{C}$. 吴家刚课 题组 ${ }^{[41]}$ 测试了 $(1-x) \mathrm{Bi}_{1.05} \mathrm{Fe}_{1-y} \mathrm{Sc}_{y} \mathrm{O}_{3}-x \mathrm{BaTiO}_{3}$ 陶瓷的铁 电相变, 实验结果表明, 当 $y=0.03$ 时, $T_{\mathrm{C}}$ 随 $x$ 从 0.25 增 加到 $0.35, T_{\mathrm{C}}$ 单调下降到 $420^{\circ} \mathrm{C}$; 当 $x=0.30$ 时, $y$ 从 0.01 增加到 $0.10, T_{\mathrm{C}}$ 从 $520^{\circ} \mathrm{C}$ 单调下降至 $460{ }^{\circ} \mathrm{C}$; 对于 $x=0.30, y=0.03$ 组分, 当 $\mathrm{Al}$ 替代 $\mathrm{Sc}$ 时居里温度降低 $\sim 30{ }^{\circ} \mathrm{C}$. Reaney课题组 ${ }^{[42]}$ 对 $(0.95-x) \mathrm{BiFeO}_{3}-x \mathrm{BaTiO}_{3}-$ $0.05 \mathrm{BiScO}_{3}$ 三元固溶体的研究结果表明, 当 $x$ 从 0.25 增加到 0.40 时, 居里温度单调降低. 赁敦敏课题组 ${ }^{[43]}$ 研究了 $1.0 \%$ (摩尔百分比) $\mathrm{Mn}$ 掺杂 $0.725 \mathrm{BiFe}_{1-x} \mathrm{Sc}_{x} \mathrm{O}_{3}$ $0.275 \mathrm{BaTiO}_{3}$ 陶瓷, 观察到 $T_{\mathrm{C}}$ 与烧结温度和 $\mathrm{Sc}$ 的含量 存在依赖现象: $x=0.02$ 的陶瓷样品, 烧结温度从 $880^{\circ} \mathrm{C}$ 升高至 $1020^{\circ} \mathrm{C}$, 居里温度 $T_{\mathrm{C}}$ 从 $660^{\circ} \mathrm{C}$ 降低到 $592^{\circ} \mathrm{C}$; 改 变 $\mathrm{Sc}$ 含量, $T_{C}$ 的变化没有单调依赖关系. 需要说明的 是, 吴家刚 ${ }^{[41]}$ 和 Reaney 课题 组 ${ }^{[42]}$ 制备的 BF-BT$\mathrm{BiScO}_{3}$ 陶瓷样品的介电损耗 $\tan \delta$ 分别为 $\sim .12 @ 100$ $\mathrm{kHz}$ 和 $\sim 0.10 @ 10 \mathrm{kHz}$; 而赁敦敏课题组 ${ }^{[4]}$ 铁电陶瓷 的 $\tan \delta \sim 0.035 @ 100 \mathrm{kHz}$.

用 $\mathrm{Bi}_{1 / 2} \mathrm{Nb}_{1 / 2}, \mathrm{Zn}_{1 / 3} \mathrm{Nb}_{2 / 3}, \mathrm{Al}_{1 / 2} \mathrm{Nb}_{1 / 2}$ 替代等摩尔量 的 $\mathrm{Ti}$, 测得 $\mathrm{Mn}$ 掺杂 $\mathrm{BF} 69-\mathrm{BT} 27-\mathrm{Ba}\left(\mathrm{Zn}_{1 / 3} \mathrm{Nb}_{2 / 3}\right) \mathrm{O}_{3}$ 铁电 陶瓷的 $T_{\mathrm{C}}=429^{\circ} \mathrm{C}, \mathrm{BF} 69-\mathrm{BT} 27-\mathrm{Ba}\left(\mathrm{Al}_{1 / 2} \mathrm{Nb}_{1 / 2}\right) \mathrm{O}_{3}$ 的 $T_{\mathrm{C}}=$ $468^{\circ} \mathrm{C}, \mathrm{BF} 69-\mathrm{BT} 27-\mathrm{Ba}\left(\mathrm{Bi}_{1 / 2} \mathrm{Nb}_{1 / 2}\right) \mathrm{O}_{3}$ 的 $T_{\mathrm{C}}=492^{\circ} \mathrm{C}$; 与 此相对应的是, Mn掺杂 BF69-BT31铁电陶瓷的 $T_{\mathrm{C}}=$ $479^{\circ} \mathrm{C}$. 对比表 $\mathrm{S} 1$ 数据, 基于前述对钙钛矿结构氧化 物铁电相变居里温度和铁电性的数据挖掘关系可知, 此处铁电相变居里温度的变化与替代原子的质量和 大小变化趋势没有单调的对应关系: 一方面, 这些替 代元素对居里温度的影响可能属于 $T_{\mathrm{C}}(\mu)=a+b \mu$ $+c \mu^{2}$ 方程不同的系数组 ${ }^{[24,25,44]}$; 另一方面, 不同课 题组实验数据之间存在较大差异, 对组分和浓度变 化的观测甚至得到相反的变化趋势, 因此需要提高 样品质量和实验数据的可靠性. 这些事实表明, 我们 不仅需要更多的实验数据和模拟计算以对它们的贡 献进行量化分析, 更需要提高样品质量(降低介电损 耗等) 来获得可靠的实验数据, 以便进行深人的数据

\section{挖掘工作.}

另一方面, 图3(a)所示结果表明, BF- $x$ BT基钙钛 矿结构固溶体氧化物在室温 $T_{\mathrm{C}}$ 范围内不存在其他结 构相变. 该特征对获得具有良好温度稳定性的铁电 压电陶瓷材料非常重要, 这是因为铁电陶瓷在经历 温度循环变化时, 铁电畴在不同态之间的转化会破 坏极化处理诱导的特定极化状态, 从而恶化与极化 状态紧密相关的各种性能参数的温度稳定性 ${ }^{[7,8]}$. 本 课题组的前期实验表明, 充分极化的BF-BT-BZT铁 电陶瓷材料具有优异的温度和时间老化稳定 性 ${ }^{[13 ~ 15,20]}$. 对不同钙钛矿结构的氧化物体系铁电陶 瓷进行数据挖掘, 结果发现: $(\mathrm{K}, \mathrm{Na}) \mathrm{NbO}_{3}$ 系铁电陶瓷 的压电响应经元素替代, $d_{33}$ 已提高到 $490 \mathrm{pC} / \mathrm{N}$; 但 $T_{\mathrm{C}}$ 以下存在铁电-铁电结构相变, 比如 $0.96\left(\mathrm{Na}_{0.52} \mathrm{~K}_{0.48}\right)$ $\left(\mathrm{Nb}_{0.95} \mathrm{Sb}_{0.05}\right) \mathrm{O}_{3}-0.04 \mathrm{Bi}_{0.5}\left(\mathrm{Na}_{0.82} \mathrm{~K}_{0.18}\right)_{0.5} \mathrm{ZrO}_{3}\left(T_{\mathrm{C}}=227^{\circ} \mathrm{C}\right)$ 铁电陶瓷在室温附近存在一个三方-四方铁电结构相 变 ${ }^{[45]}$; 准同型结构相界附近组分的 $\left(\mathrm{Bi}_{0.5}(\mathrm{Na}, \mathrm{K})_{0.5}\right) \mathrm{TiO}_{3}-$ $\mathrm{BaTiO}_{3}$ 陶瓷在 $100^{\circ} \mathrm{C} \sim T_{\mathrm{C}}$ 范围内存在一个铁电-反铁电 结构相变；而 $\mathrm{Ba}\left(\mathrm{Zr}_{0.2} \mathrm{Ti}_{0.8}\right) \mathrm{O}_{3}-\left(\mathrm{Ba}_{0.7} \mathrm{Ca}_{0.3}\right) \mathrm{TiO}_{3}$ 陶瓷的 三方-四方铁电结构相变温度恰巧在室温附近. 与此 形成鲜明对比的是, 在准同型结构相界四方相侧, 包 括温度稳定性在内的具有优异综合性能的PZT铁电 陶瓷在室温 $T_{C}$ 范围内不存在任何结构相变 ${ }^{[8]}$.

对不同元素替代BF- $x$ BT铁电陶瓷材料进行极化 处理, 设定的极化条件为在 $120^{\circ} \mathrm{C}$ 硅油中加 $8 \mathrm{kV} / \mathrm{mm}$ 的直流电场保压 $15 \mathrm{~min}$; 极化后样品放置 $24 \mathrm{~h}$, 测试 压电常数 $d_{33} .1 \mathrm{kHz}$ 频率的介电常数 $\varepsilon_{33}{ }^{\mathrm{T}} / \varepsilon_{0}$ 和介电损 耗 $\tan \delta$, 极化情况和所得结果总结于表 $\mathrm{S} 1$. 从表 $\mathrm{S} 1$ 可 以看到, 不同的元素替代需要不同的烧结条件以获 得较低的介电损耗，而不同元素替代也表现出不同 的耐压特性. 比如, $920^{\circ} \mathrm{C}$ 烧结 $5 \mathrm{~h}$ 的 $0.8 \%$ (摩尔百分 比) Mn 掺杂BF69-BT27-BiScO 3 铁电陶瓷, 介电损耗 $\tan \delta=0.087$ 较大, 无法在 $120^{\circ} \mathrm{C}$ 高温极化; 室温 8 $\mathrm{kV} / \mathrm{mm}$ 场强极化 $15 \mathrm{~min}$ 后, 实验测得 $\varepsilon_{33}{ }^{\mathrm{T}} / \varepsilon_{0}=541$, $\tan \delta=0.142, d_{33}=39 \mathrm{pC} / \mathrm{N} ; 930^{\circ} \mathrm{C}$ 烧结 $5 \mathrm{~h}$ 的 $0.6 \%$ (摩尔 百分比) Mn掺杂BF69-BT27-BiAlO 陶瓷, 介电损耗 $\tan \delta=0.045$ 虽然已经大幅降低, 仍然无法在 $120^{\circ} \mathrm{C}$ 高 温极化. 室温7.4 $\mathrm{kV} / \mathrm{mm}$ 场强时击穿, 测得 $\varepsilon_{33}{ }^{\mathrm{T}} / \varepsilon_{0}=$ $510, \tan \delta=0.049, d_{33}=28 \mathrm{pC} / \mathrm{N} ; 930^{\circ} \mathrm{C}$ 烧结7 h $0.6 \%$ (摩尔 百分比)Mn掺杂BF69-BT27- $\mathrm{Ba}\left(\mathrm{Bi}_{1 / 2} \mathrm{Nb}_{1 / 2}\right) \mathrm{O}_{3}$ 陶瓷, $\tan \delta=$ $0.059,120^{\circ} \mathrm{C}$ 高温, $7 \mathrm{kV} / \mathrm{mm}$ 场强极化 $15 \mathrm{~min}, \varepsilon_{33}{ }^{\mathrm{T}} / \varepsilon_{0}=$ $477, \tan \delta=0.049, d_{33}=32 \mathrm{pC} / \mathrm{N}$; 对于 $0.6 \%$ (摩尔百分比) 
$\mathrm{Mn}$ 掺杂 BF69-BT27- $\mathrm{BaBO}_{3}\left(\mathrm{~B}=\mathrm{Al}_{1 / 2} \mathrm{Nb}_{1 / 2}, \mathrm{Zn}_{1 / 3} \mathrm{Nb}_{2 / 3}\right.$, $\mathrm{Zr}$ 和 $\mathrm{Sn}$ ) 陶瓷, 即使在室温也无法加高场强进行极化 处理. 吴家刚课题组 ${ }^{[41]}$ 对具有较大介电损耗的 $(1-x) \mathrm{Bi}_{1.05} \mathrm{Fe}_{1-y} \mathrm{~A}_{y} \mathrm{O}_{3}-x \mathrm{BaTiO}_{3}(\mathrm{~A}=\mathrm{Sc}, \mathrm{Ga}, \mathrm{Al}$ 等; 0.20 $\leqslant x \leqslant 0.35 / y \leqslant 0.10)$ 铁电陶瓷, $120^{\circ} \mathrm{C}$ 高温, $5 \mathrm{kV} / \mathrm{mm}$ 场 强极化处理 $30 \mathrm{~min}$, 观测到的压电响应 $d_{33} \leqslant 180$ $\mathrm{pC} / \mathrm{N}$. 赁敦敏课题组 ${ }^{[43]}$ 对具有较小介电损耗 $(\tan \delta \sim$ $0.035)$ 的 $\mathrm{Mn}$ 掺杂 $0.725 \mathrm{BiFe}_{1-x} \mathrm{Sc}_{x} \mathrm{O}_{3}-0.275 \mathrm{BaTiO}_{3}(x \leqslant$ $0.08)$ 陶瓷采用的极化程序是, $120 \sim 150^{\circ} \mathrm{C}$ 极化 $20 \mathrm{~min}$, 然后在 $6 \mathrm{kV} / \mathrm{mm}$ 场强下冷却到室温, 观测到的压电响 应 $d_{33} \leqslant 129 \mathrm{pC} / \mathrm{N}$. 到目前为止, 在已尝试过的各种 第三组元中, BF- $x$ BT- $y$ BZT三元固溶体陶瓷具有最好 的耐压特性, $120^{\circ} \mathrm{C}$ 高温击穿场强大于 $8 \mathrm{kV} / \mathrm{mm}$. 比 如, $920^{\circ} \mathrm{C}$ 烧结 $10 \mathrm{~h}, 1.1 \%$ (摩尔百分比) Mn掺杂BF65BT32-BZT3 陶瓷极化后的压电性能为 $\varepsilon_{33}{ }^{\mathrm{T}} / \varepsilon_{0}=650$, $\tan \delta=0.048, d_{33}=127 \mathrm{pC} / \mathrm{N} ; 930^{\circ} \mathrm{C}$ 烧结 $5 \mathrm{~h}, 0.8 \%$ (摩尔 百分比)Mn掺杂BF74-BT22-BZT4陶瓷极化后的压电 性能为 $\varepsilon_{33}{ }^{\mathrm{T}} / \varepsilon_{0}=274, \tan \delta=0.012, d_{33}=75 \mathrm{pC} / \mathrm{N}$; 更多例 子见本课题组前期工作报道 ${ }^{[13 \sim 15,37]}$.

铁电唯象理论表明, 压电响应 $d_{33}$ 与电致伸缩系 数 $Q_{11}$ 、诱导极化强度 $P_{3}$ 、介电常数 $\varepsilon_{33}$ 满足 $d_{33}=$ $2 Q_{11} P_{3} \varepsilon_{33}{ }^{[1]}$. 根据电介质物理理论, 诱导极化强度 $P_{3}$ 可以用介电常数 $\varepsilon_{33}$ 表示. 由此, 上述压电响应关系 通过模式识别之主成分分析法 (principal component analysis) 降维转化为 $d_{33}=a \varepsilon_{33}-b \varepsilon_{33}^{2}$ 形式, 式中的系 数 $(a, b)$ 不是常数、与电致伸缩系数等物理量相 关 ${ }^{[13 ~ 15,38]}$. 在Origin软件包中对钙钛矿等结构铁电陶 瓷的 $d_{33}$ 与 $\varepsilon_{33}$ 关系进行最小二乘法拟合, 它们满足 $d_{33}=0.24 \varepsilon_{33}-0.000018 \varepsilon_{33}^{2}$ 统计关系 ${ }^{[38,39]}$. 压电常数和 介电常数之间的强相关性源于, 它们都是铁电自发 极化同一物理量对不同外场的响应特性. 通过数据 挖掘得到了压电响应和介电常数之间量化关联关 系 ${ }^{[13 \sim 15,20,37 \sim 39]}$, 它表明要想获得大的压电响应、需要 有效提高介电常数. 进一步的数据挖掘可知, 铁电材 料组成元素的极化能力、浓度是否在晶体结构相界附 近是获得高介电常数(压电响应)的本征因素, 而缺陷 种类与浓度、陶瓷材料微结构(包括致密度、晶界、 晶粒大小及其分布) 等是调控介电常数(压电响应)的 非本征因素; 另一方面它也可以用作铁电陶瓷极化 处理是否饱和的判据 ${ }^{[13,20]}$.

$\mathrm{BF}-x \mathrm{BT}$ 系铁电陶瓷压电响应性能与降低陶瓷介 电损耗的研究紧密相关. Leontsev和Eitel ${ }^{[9]}$ 采用 $0.1 \%$
(重量百分比) $\mathrm{MnO}_{2}$ 掺杂和优化烧结条件, 在降低介 电损耗的基础上实现了 $\mathrm{BF}-x \mathrm{BT}(x=0.25 \sim 0.33)$ 铁电陶 瓷的极化: $x=0.25$ 时介电损耗 $\tan \delta$ 降低至 0.046 , 获得 的较大压电响应 $d_{33}=116 \mathrm{pC} / \mathrm{N}$. 程晋荣课题组 ${ }^{[31]}$ 对不 同组分BF- $x$ BT陶瓷进行的研究, 结果表明 $1.0 \%$ (摩尔 百分比) $\mathrm{Mn}$ 掺杂 $\mathrm{BF}-0.3 \mathrm{BT}$ 铁电陶瓷具有较大压电响 应: $d_{33}=177 \mathrm{pC} / \mathrm{N}, \varepsilon_{\mathrm{r}}=740, \tan \delta=0.045$ (从对应的介电 常数 $\varepsilon_{r}$ 计算 $\left.d_{33}=167 \mathrm{pC} / \mathrm{N}\right)$; 结构测试显示该组分为三 方-赝立方结构相界. 赁敦敏课题组 ${ }^{[12]}$ 同时掺杂 $\mathrm{Mn}$, 加人第三组元和优化烧结条件, $(0.70-y)$ BF-0.30BT$y \operatorname{BZT}(y=0.00 \sim 0.15)$ 陶瓷的 $\tan \delta<0.047$, 在 $y=0.05$ 时获 得极大压电响应 $d_{33}=139 \mathrm{pC} / \mathrm{N} .2015$ 年, Song课题 组 ${ }^{[46]}$ 报道了 $0.97\left[0.67 \mathrm{Bi}_{1.05} \mathrm{FeO}_{3}-0.33 \mathrm{BaTiO}_{3}\right]-0.03 \mathrm{Bi}_{1.05}$ $\left(\mathrm{Zn}_{0.5} \mathrm{Ti}_{0.5}\right) \mathrm{O}_{3}$ 铁电陶瓷具有可观的压电响应性能 $\left(d_{33}=\right.$ $\left.324 \mathrm{pC} / \mathrm{N}, T_{\mathrm{C}}=466^{\circ} \mathrm{C}\right)$; 然而, 对工程应用至关重要的 介电常数和介电损耗性质参数没有明确给出. 近年 来, 多个课题组围绕此组分进行了重复实验, 遗憾的 是, 压电响应远低于Song课题组 ${ }^{[46]}$ 报道的数值. 吴 家刚课题组 ${ }^{[47]}$ 研究了不同组分 $(1-y)[(1-x) \mathrm{BF}-x \mathrm{BT}]-$ $y \mathrm{BZT}(0.25 \leqslant x \leqslant 0.35 / 0.01 \leqslant y \leqslant 0.05)$ 陶瓷的压电性能: 当 $y=0.03$ 时, 压电响应在 $x=0.285$ 时具有极大值 $d_{33}=$ $155 \mathrm{pC} / \mathrm{N}$; 当 $x=0.285$ 时, 压电响应在 $y=0.01$ 时具有极 大值 $d_{33}=195 \mathrm{pC} / \mathrm{N}$ (从对应的介电常数 $\varepsilon_{r}$ 计算得到 $\left.d_{33}=159 \mathrm{pC} / \mathrm{N}\right)$; 当 $x=0.33, y=0.03$ 时, 陶瓷的 $\varepsilon_{r} \sim 710$, $\tan \delta \sim 0.122, T_{\mathrm{C}} \sim 420^{\circ} \mathrm{C}, d_{33} \sim 110 \mathrm{pC} / \mathrm{N}$, 压电响应低于 从介电常数 $\varepsilon_{r} \sim 710$ 计算得到的 $d_{33}$ 值. 张波萍课题组 ${ }^{[48]}$ 在 $0.7 \mathrm{BiFe}_{0.95}\left(\mathrm{Zn}_{0.5} \mathrm{Ti}_{0.5}\right)_{0.05} \mathrm{O}_{3}-0.3 \mathrm{BaTiO}_{3}$ 组分获得了 较大压电响应 $d_{33}=135 \mathrm{pC} / \mathrm{N}$, 此时, $\tan \delta=0.07, T_{\mathrm{C}}=$ $482^{\circ} \mathrm{C}$. 反观PZT铁电压电陶瓷材料的研究历史, 获 得工程应用要求水平的介电损耗性质是比较压电响 应性能的前提和基础.

不同元素替代BF- $x$ BT 铁电陶瓷的压电常数 $d_{33}$ 、 介电常数 $\varepsilon_{33}$ 和居里温度 $T_{\mathrm{C}}$ 以原胞约合质量 $\mu$ 为横坐 标作图, 结果见图 4. 为了对比, 用 $d_{33}=0.24 \varepsilon_{33}-$ $0.000018 \varepsilon_{33}^{2}$ 计算得到的压电响应 $d_{33}$ 值也放于图 4. 结 果表明, 具有较低介电损耗的BF-BT-BZT三元固溶 体铁电陶瓷的压电响应 $d_{33}$ 实验值高于从 $\varepsilon_{33}$ 得到的计 算预测值. 如图4所示, 程晋荣课题组对具有较低介 电损耗的 BF- $x \mathrm{BT}$ 二元固溶体铁电陶瓷的实验结果也 符合该预测关系.

对于前期优化获得的 $0.17 \%$ (重量百分比) $\mathrm{MnO}_{2}$ 掺杂BF69-BT27-BZT4铁电陶瓷, $d_{33}=145 \mathrm{pC} / \mathrm{N}$, 


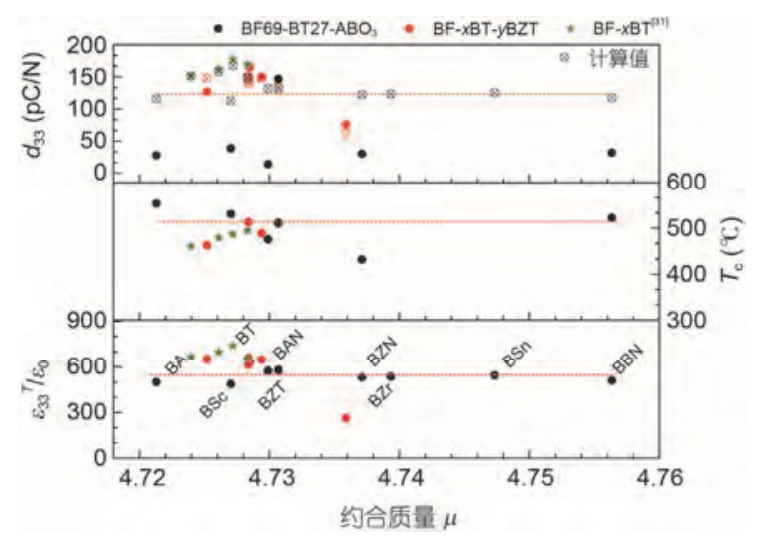

图 4 (网络版彩色) 不同元素替代BF- $x$ BT陶瓷的压电常数 $d_{33}$, 介电常 数 $\varepsilon_{33}$ 和居里温度 $T_{\mathrm{C}}$ 与钙钟矿结构氧化物的原胞约合质量 $(\mu)$ 的关系. $\mathrm{BF} 69-\mathrm{BT} 27-\mathrm{BaBO}_{3}(\mathrm{~B}=\mathrm{Zr}$ 和 $\mathrm{Sn})$ 陶瓷的介电常数 $\mathcal{E}_{\mathrm{r}}$ 由介电频谱测量外 推得到. 为了对比, BF- $x \mathrm{BT}^{[31]}$ 和BF- $x \mathrm{BT}-y \mathrm{BZT}$ 铁电压电陶瓷的结果 也在图中一并给出, 后者的部分结果见文献[13 15]. 图中虚线是为 了方便视图

Figure 4 (Color online) Plotting of piezoelectric constant $d_{33}$, dielectric constant $\varepsilon_{33}$ and Curie temperatures $T_{\mathrm{C}}$ with respect to reduced mass of perovskite unit cell $(\mu)$ for various BF- $x \mathrm{BT}$-based ternary solid solution ceramics. The values for $\mathrm{BF} 69-\mathrm{BT} 27-\mathrm{BaBO}_{3}(\mathrm{~B}=\mathrm{Zr}$ and $\mathrm{Sn})$ ceramics were obtained through extrapolation of frequency-dependent dielectric spectrum. For comparison, some results for BF- $x \mathrm{BT}^{[31]}$ and BF- $x \mathrm{BT}-$ $y$ BZT (this work and Refs. [13-15]) ferroelectric piezoceramics were also presented together. Dashed line is only for view

$\varepsilon_{33}{ }^{\mathrm{T}} / \varepsilon_{0}=570, \tan \delta=0.034$. 一方面, 进一步优化 $\mathrm{Mn}$ 掺 杂量和烧结条件, $0.7 \%$ (摩尔百分比) Mn掺杂 BF69BT27-BZT4铁电陶瓷的压电性能为 $d_{33}=133 \mathrm{pC} / \mathrm{N}$, $\varepsilon_{33}{ }^{\mathrm{T}} / \varepsilon_{0}=550$ 和 $\tan \delta=0.025$. 这不仅是 BF- $x \mathrm{BT}$ 系铁电陶 瓷介电损耗性质首次达到工程应用水平, 也初步反 映了压电响应与介电损耗两个参数之间存在相关性: 都与陶瓷材料的缺陷状态紧密相关.

另一方面, 为探索 $\mathrm{A}$ 位离子的影响, 初步进行了 2 个组分实验: (1) 分别加 $0.3 \%$ (摩尔百分比)的 $\mathrm{SrTiO}_{3}$ 和 $0.3 \%$ (摩尔百分比) 的 $\mathrm{PbTiO}_{3}, 910{ }^{\circ} \mathrm{C}$ 烧结 $10 \mathrm{~h}$, $120^{\circ} \mathrm{C}, 7 \mathrm{kV} / \mathrm{mm}$ 场强下, 极化 $15 \mathrm{~min}$, 测得陶瓷性能 为 $\varepsilon_{33}{ }^{\mathrm{T}} / \varepsilon_{0}=613, \tan \delta=0.033, d_{33}=144 \mathrm{pC} / \mathrm{N} ;$ (2) 外加 $0.3 \%$ (摩尔百分比)的 $\mathrm{SrTiO}_{3}$ 和 $2.3 \%$ (摩尔百分比)的 $\mathrm{PbTiO}_{3}, 910^{\circ} \mathrm{C}$ 烧结 $10 \mathrm{~h}, 120^{\circ} \mathrm{C}, 7 \mathrm{kV} / \mathrm{mm}$, 极化 15 $\min$, 性能为 $\varepsilon_{33}{ }^{\mathrm{T}} / \varepsilon_{0}=649, \tan \delta=0.035, d_{33}=150 \mathrm{pC} / \mathrm{N}$, 压电响应高于从介电常数 $\varepsilon_{33}{ }^{\mathrm{T}} / \varepsilon_{0}=649$ 计算得到的 $d_{33}(148 \mathrm{pC} / \mathrm{N})$ 值; $120^{\circ} \mathrm{C}, 8 \mathrm{kV} / \mathrm{mm}$ 场强下极化 $15 \mathrm{~min}$, 性能为 $\varepsilon_{33}{ }^{\mathrm{T}} / \varepsilon_{0}=657, \tan \delta=0.035, d_{33}=130 \mathrm{pC} / \mathrm{N}$. 与 $\mathrm{PZT}$ 铁电压电陶瓷相似, 当对BF- $x$ BT系铁电陶瓷进行不 同的元素替代和掺杂等改性后, 需要进行相应的烧 结条件和极化条件优化.
基于压电响应与介电性质的材料信息学相关性, 高性能铁电压电陶瓷新材料的探索模式与传统试错 模式有很大的不同, 我们可以把压电响应性能的直 接探索简化为对介电性质的探索和压电性能的验证 两个步骤来完成. 这样可以带来3个方面的优势: (1) 省去很多铁电陶瓷的高温极化处理工作. 极化处理 的成效与陶瓷样品的直流电阻(离子迁移)和介电损 耗(缺陷种类和浓度)这两个性质紧密相关(表 $S 1$ ), 成 功的极化处理需要耗时费力的样品制备工艺条件的 优化工作; (2) 对那些未掺杂、未优化工艺条件获得 的介电损耗较大的样品, 我们能够通过介电性质的 测量初步预测压电响应性能水平, 即从失败的实验 获得隐藏的材料性能信息 ${ }^{[49]}$. 例如, 表 S1所示 $0.6 \%$ (摩尔百分比) $\mathrm{Mn}$ 掺杂 $\mathrm{BF} 69-\mathrm{BT} 27-\mathrm{BaBO}_{3}(\mathrm{~B}=\mathrm{Zr}$ 和 $\mathrm{Sn}$ ) 陶瓷的介电损耗较大 $(\tan \delta>1.1)$, 从介电频谱测试和 电介质理论分析可知，此时介电损耗包含空间电荷 和铁电极化弛豫两部分贡献 ${ }^{[50]}$ : 空间电荷的贡献主 要在 $10 \mathrm{kHz}$ 频率以下, 从频谱的高频部分可以线性 外推 $1 \mathrm{kHz}$ 时铁电极化贡献部分的介电常数 $\varepsilon_{r}$ 分别为 536 和 544 , 以此介电常数值估算的压电响应 $d_{33}$ 分别为 123 和 $125 \mathrm{pC} / \mathrm{N}$. 通过调控 Mn掺杂量和优化工艺条 件, 本课题组对多种组分饱和极化样品的实验测量 值与外推值的偏差<5\%; (3) 在庞大的钙钛矿结构氧 化物化学组成空间, 可以采用机器学习和第一性原 理计算等方法进行组分節选、介电性能计算预测, 降 低实验篮选的工作量, 实现新材料的快速发现 ${ }^{[51]}$.

在考虑工程应用要求的介电损耗性质和极化饱 和前提下, 本文从 BF-BT-BZT三元固溶体中优选出 两个组分的钙钛矿型高性能高温压电陶瓷材料: (1) $\mathrm{Mn}$ 掺杂BF74-BT22-BZT4, 综合压电性能为 $d_{33} \sim 75$ $\mathrm{pC} / \mathrm{N}, \varepsilon_{33}{ }^{\mathrm{T}} / \varepsilon_{0} \sim 260, \tan \delta \sim 0.01, k_{t} \sim 0.42, k_{p} \sim 0.29, T_{\mathrm{C}} \sim$ $630{ }^{\circ} \mathrm{C}$, 优于目前商用铋层状钛酸铋系压电陶瓷 $\left(\mathrm{K}-15: d_{33}=18 \mathrm{pC} / \mathrm{N}, \varepsilon_{33}{ }^{\mathrm{T}} / \varepsilon_{0}=140, \tan \delta=0.03, k_{t}=0.23\right.$, $k_{p}=0.025, T_{\mathrm{C}}=600^{\circ} \mathrm{C}$ ); (2) $\mathrm{Mn}$ 掺杂 $\mathrm{BF} 69-\mathrm{BZT} 4-\mathrm{BT} 27$, 综合压电性能为 $d_{33} \sim 145 \mathrm{pC} / \mathrm{N}, \varepsilon_{33}{ }^{\mathrm{T}} / \varepsilon_{0} \sim 570, \tan \delta \sim$ $0.03, k_{t} \sim 0.41, k_{p} \sim 0.33, T_{\mathrm{C}} \sim 510^{\circ} \mathrm{C}$, 优于目前铇青铜结 构偏铌酸铅商用压电陶瓷 $\left(\mathrm{K}-81: d_{33}=85 \mathrm{pC} / \mathrm{N}\right.$, $\varepsilon_{33}{ }^{\mathrm{T}} / \varepsilon_{0}=300, \tan \delta=0.01, k_{t}=0.33, k_{p}=0.04, T_{\mathrm{C}}=460^{\circ} \mathrm{C}$ ). 与偏铌酸铅和钛酸铋系压电陶瓷材料相比, 钙钛矿 型BF-BT-BZT固溶体压电陶瓷材料没有结构各向异 性、没有压电性能的各向异性, 可研发切变式高温压 电加速度传感器. 需要指出的是, BF69-BZT4-BT27 
无铅压电陶瓷的性能也与 PZT-2含铅压电陶瓷材料 的性能相当. 由此可见, 基于材料信息学的数据挖掘 是发现数据隐含的信息和规律、加速新材料研发的有 效工具, 借鉴 KNN和PZT系铁电陶瓷压电响应从二 元到三元、再到多元固溶体的进化史 ${ }^{[1,45]}$, 通过新数 据的不断生产和深人数据挖掘, 必将为下一步高稳 定性的 $d_{33}>300 \mathrm{pC} / \mathrm{N}, T_{\mathrm{C}}>300^{\circ} \mathrm{C}$ 的无铅压电陶瓷新材 料的发现提供强力的方向性指导.

\section{3 小结}

有别于传统的试错式, 本文在钙钛矿结构氧化 物铁电材料数据挖掘结果的指导下, 从离子质量的
轻重、半径的大小出发选择不同组合特征的元素，对 $\mathrm{BF}-x \mathrm{BT}$ 固溶体陶瓷进行替代实验, 重点研究了三方赝立方结构相界附近组分的 BF- $x$ BT 基三元固溶体陶 瓷的铁电相变和介电、压电响应性能. 实验发现：替 代元素的性质是决定陶瓷介电损耗和压电响应的重 要因素; 在Mn掺杂BF-BT-BZT三元固溶体中获得了 工程应用需求水平的介电损耗性质, 从中篮选出两 个组分的钙钛矿型高性能高温铁电压电陶瓷新材料, 它们的压电响应性能 $\left(d_{33}\right)$ 分别是商用偏铌酸铅和钛 酸铋系压电陶瓷材料的 1.5 和 4.1 倍, 且可采用传统电 子陶瓷工艺规模生产, 是高灵敏高温压电传感器工 程应用的新材料选项。

\section{参考文献}

1 Haertling G H. Ferroelectric ceramics: History and technology. J Am Ceram Soc, 1999, 82: 797-818

2 Stevenson T, Martin D G, Cowin P I, et al. Piezoelectric materials for high temperature transducers and actuators. J Mater Sci Mater Electron, 2015, 26: 9256-9267

3 Saito Y, Takao H, Tani T, et al. Lead-free piezoceramics. Nature, 2004, 432: 84-87

4 Takenaka T, Maruyama K, Sakata K. $\left(\mathrm{Bi}_{1 / 2} \mathrm{Na}_{1 / 2}\right) \mathrm{TiO}_{3}-\mathrm{BaTiO}_{3}$ system for lead-free piezoelectric ceramics. Jpn J Appl Phys, 1991, 30: 2236-2239

5 Liu W, Ren X B. Piezoelectric effect in Pb-free ceramics. Phys Rev Lett, 2009, 103: 257602

6 Bao H X, Zhou C, Xue D Z, et al. A modified lead-free piezoelectric BZT- $x$ BCT system with higher $T_{\mathrm{c}}$. J Phys D Appl Phys, 2010, 43 : 465401

7 Shrout T R, Zhang S J. Lead-free piezoelectric ceramics: Alternatives for PZT? J Electroceram, 2007, 19: 113-126

8 Rödel J, Jo W, Seifert K T P, et al. Perspective on the development of lead-free piezoceramics. J Am Ceram Soc, 2005, 92: 1153-1177

9 Leontsev S O, Eitel R E. Dielectric and piezoelectric properties in Mn-modified $(1-x) \mathrm{BiFeO}_{3}-x \mathrm{BaTiO}_{3}$ ceramics. J Am Ceram Soc, 2009, 92: $2957-2961$

10 Zhang L L, Yu J. Robust insulating La and Ti co-doped $\mathrm{BiFeO}_{3}$ multiferroic ceramics. J Mater Sci Mater Electron, 2016, 27: 8725-8733

11 Shan X, Zhou C R, Cen Z Y, et al. $\mathrm{Bi}\left(\mathrm{Zn}_{1 / 2} \mathrm{Ti}_{1 / 2}\right) \mathrm{O}_{3}$ modified $\mathrm{BiFeO}_{3}-\mathrm{BaTiO}_{3}$ lead-free piezoelectric ceramics with high temperature stability. Ceram Int, 2013, 39: 6707-6712

12 Zheng Q J, Guo Y Q, Lei F Y, et al. Microstructure, ferroelectric, piezoelectric and ferromagnetic properties of $\mathrm{BiFeO}_{3}-\mathrm{BaTiO}_{3}-\mathrm{Bi}$ $\left(\mathrm{Zn}_{0.5} \mathrm{Ti}_{0.5}\right) \mathrm{O}_{3}$ lead free multiferroic ceramics. J Mater Sci Mater Electron, 2014, 25: 2638-2648

13 Lin Y, Zhang L L, Zheng W L, et al. Structural phase boundary of $\mathrm{BiFeO}_{3}-\mathrm{Bi}\left(\mathrm{Zn}_{1 / 2} \mathrm{Ti}_{1 / 2}\right) \mathrm{O}_{3}-\mathrm{BaTiO}_{3}$ lead-free ceramics and their piezoelectric properties. J Mater Sci Mater Electron, 2015, 26: 7351-7360

14 Lin Y, Zhang L L, Yu J. Stable piezoelectric property of modified $\mathrm{BiFeO}_{3}-\mathrm{BaTiO}_{3}$ lead-free piezoceramics. J Mater Sci Mater Electron, 2015, 26: 8432-8441

15 Lin Y, Zhang L L, Yu J. Piezoelectric and ferroelectric property in Mn-doped $0.69 \mathrm{BiFeO}_{3}-0.04 \mathrm{Bi}\left(\mathrm{Zn}_{1 / 2} \mathrm{Ti}_{1 / 2}\right) \mathrm{O}_{3}-0.27 \mathrm{BaTiO}{ }_{3}$ lead-free piezoceramics. J Mater Sci Mater Electron, 2016, 27: 1955-1965

16 Agrawal A, Choudhary A. Perspective: Materials informatics and big data: Realization of the "fourth paradigm" of science in materials science. APL Mater, 2016, 4: 053208

17 Ramprasad R, Batra R, Pilania G, et al. Machine learning in materials informatics: Recent applications and prospects. NPJ Comput Mater, 2017, 3: 54

18 Wagner N, Rondinelli J M. Theory-guided machine learning in materials science. Front Mater, 2016, 3: 28

19 Ghiringhelli L M, Vybiral J, Levchenko S V, et al. Big data of materials science: Critical role of the descriptor. Phys Rev Lett, 2015, 114: 105503 
20 Yu J, Zhang L L, Hou X B, et al. Novel perovskite-type ferroelectrics with high curie temperature and piezoresponse. IEEE Xplore, 2016, doi: 10.1109/ISAF.2016.7578087

21 Anderson P W. More is different: Broken symmetry and the nature of the hierarchical structure of science. Science, 1972, 177: 393-396

22 Lines M E, Glass A M. Principles and Applications of Ferroelectrics and Related Materials. New York: Clarendon Press, 1977

23 Morf R, Schneider T, Stoll E. Nonuniversal critical behavior and its suppression by quantum fluctuations. Phys Rev B, 1977, 16: 462-469

24 Zhang L L, Yu J, Itoh M. Structural phase transitions of robust insulating $\mathrm{Bi}_{1-x} \mathrm{La}_{x} \mathrm{Fe}_{1-y} \mathrm{Ti}_{y} \mathrm{O}_{3}$ multiferroics. J Appl Phys, 2014, 115: 123523

$25 \mathrm{Yu}$ J, An F F, Cao F. Ferroic phase transition of tetragonal $\mathrm{Pb}_{0.6-x} \mathrm{Ca}_{x} \mathrm{Bi}_{0.4}\left(\mathrm{Ti}_{0.75} \mathrm{Zn}_{0.15} \mathrm{Fe}_{0.1}\right) \mathrm{O}_{3}$ ceramics: Factors determining Curie temperature. Jpn J Appl Phys, 2014, 53: 051501

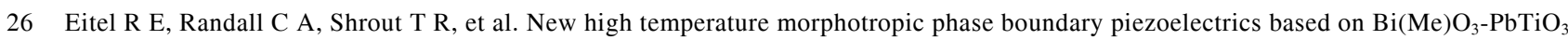
ceramics. Jpn J Appl Phys, 2001, 40: 5999-6002

27 Grinberg I, Suchomel M R, Davies P K, et al. Predicting morphotropic phase boundary locations and transition temperatures in Pb- and Bi-based perovskite solid solutions from crystal chemical data and first-principles calculations. J Appl Phys, 2005, 98 : 094111

28 Abrahams S C, Kurtz S K, Jamieson P B. Atomic displacement relationship to Curie temperature and spontaneous polarization in displacive ferroelectrics. Phys Rev, 1968, 172: 551-553

29 Grinberg I, Rappe A M. Nonmonotonic $T_{\mathrm{C}}$ trends in Bi-based ferroelectric perovskite solid solutions. Phys Rev Lett, 2007, 98 : 037603

30 Balachandran P V, Shearman T, Theiler J, et al. Predicting displacements of octahedral cations in ferroelectric perovskites using machine learning. Acta Cryst B, 2017, 73: 962-967

31 Wei J X, Fu D Y, Cheng J R, et al. Temperature dependence of the dielectric and piezoelectric properties of $x \mathrm{BiFeO}_{3}-(1-x) \mathrm{BaTiO}_{3}$ ceramics near the morphotropic phase boundary. J Mater Sci, 2017, 52: 10726-10737

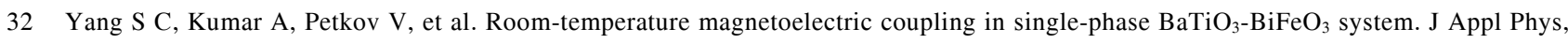
2013, 113: 144101

33 Kumar M M, Srinivas A, Suryanarayana S V. Structure property relations in $\mathrm{BiFeO}_{3} / \mathrm{BaTiO}_{3}$ solid solutions. J Appl Phys, 2000, 87: $855-862$

34 Wei Y X, Wang X T, Zhu J T, et al. Dielectric, ferroelectric, and piezoelectric properties of $\mathrm{BiFeO}_{3}-\mathrm{BaTiO}_{3}$ ceramics. J Am Ceram Soc, 2013, 96: 3163-3168

35 Randall C A, Kim N C, Kucera J P, et al. Intrinsic and extrinsic size effects in fine-grained morphotropic-phase-boundary lead zirconate titanate ceramics. J Am Ceram Soc, 1998, 81: 677-688

36 Kamel T M, de With G. Grain size effect on the poling of soft $\mathrm{Pb}(\mathrm{Zr}, \mathrm{Ti}) \mathrm{O}_{3}$ ferroelectric ceramics. J Eur Ceram Soc, 2008, 28: 851-861

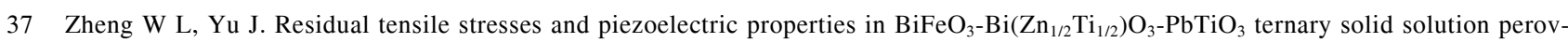
skite ceramics. AIP Adv, 2016, 6: 085314

38 Zhang L L, Hou X B, Yu J. Ferroelectric and piezoelectric properties of high temperature (Bi,La)FeO ${ }_{3}-\mathrm{Bi}_{(}\left(\mathrm{Zn}_{1 / 2} \mathrm{Ti}_{1 / 2}\right) \mathrm{O}_{3}-\mathrm{PbTiO}_{3}$ ceramics at rhombohedral/tetragonal coexistent phase. Jpn J Appl Phys, 2015, 54: 081501

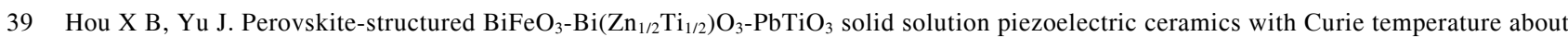
$700^{\circ} \mathrm{C}$. J Am Ceram Soc, 2013, 96: 2218-2224

$40 \mathrm{Li} \mathrm{Q}$, Wei J X, Cheng J R, et al. High temperature dielectric, ferroelectric and piezoelectric properties of $\mathrm{Mn}_{-}-\mathrm{modified}_{\mathrm{BiFeO}}-\mathrm{BaTiO}_{3}$ leadfree ceramics. J Mater Sci, 2017, 52: 229-237

41 Zheng T, Jiang Z G, Wu J G. Enhanced piezoelectricity in $(1-x) \mathrm{Bi}_{1.05} \mathrm{Fe}_{1-y} \mathrm{~A}_{y} \mathrm{O}_{3}-x \mathrm{BaTiO}_{3}$ lead-free ceramics: Site engineering and wide phase boundary region. Dalton Trans, 2016, 45: 11277-11285

42 Murakami S, Ahmed N T A F, Wang D W, et al. Optimising dopants and properties in $\mathrm{BiMeO}_{3}\left(\mathrm{Me}=\mathrm{Al}, \mathrm{Ga}, \mathrm{Sc}, \mathrm{Y}, \mathrm{Mg}_{2 / 3} \mathrm{Nb}_{1 / 3}\right.$, $\mathrm{Zn}_{2 / 3} \mathrm{Nb}_{1 / 3}, \mathrm{Zn}_{1 / 2} \mathrm{Ti}_{1 / 2}$ ) lead-free $\mathrm{BaTiO}_{3}-\mathrm{BiFeO}_{3}$ based ceramics for actuator applications. J Eur Ceram Soc, 2018, 38: 4220-4231

43 Wan Y, Li Y, Li Q, et al. Microstructure, ferroelectric, piezoelectric and ferromagnetic properties of Sc-modified $\mathrm{BiFeO}_{3}-\mathrm{BaTiO}_{3} \mathrm{mul}_{-}$ tiferroic ceramic with $\mathrm{MnO}_{2}$ addition. J Am Ceram Soc, 2014, 97: 1809-1818

44 Stringer C J, Shrout T R, Randall C A, et al. Classification of transition temperature behavior in ferroelectric $\mathrm{PbTiO}_{3}-\mathrm{Bi}\left(\mathrm{Me}^{\prime} \mathrm{Me} \mathrm{e}^{\prime \prime}\right) \mathrm{O}_{3}$ solid solutions. J Appl Phys, 2006, 99: 024106

45 Wang X, Wu J, Xiao D, et al. Giant piezoelectricity in potassium-sodium niobate lead-lree ceramics. J Am Chem Soc, 2014, 136: 2905-2910

46 Lee M H, Kim D J, Park J S, et al. High-performance lead-free piezoceramics with high curie temperatures. Adv Mater, 2015, 27: 6976-6982

47 Liu Z, Zheng T, Zhao C L, et al. Composition design and electrical properties in $\mathrm{BiFeO}_{3}-\mathrm{BaTiO}_{3}-\mathrm{Bi}_{(}\left(\mathrm{Zn}_{0.5} \mathrm{Ti}_{0.5}\right) \mathrm{O}_{3}$ lead-free ceramics. J Mater Sci Mater Electron, 2017, 28: 13076-13083 
48 Chen J Y, Zhang B P, Zhu L F, et al. Enhanced insulation resistance and electrical properties of $\mathrm{BiFe}_{1-x}\left(\mathrm{Zn}_{0.5} \mathrm{Ti}_{0.5}\right)_{x} \mathrm{O}_{3}-\mathrm{BaTiO}_{3}$ lead-free piezoceramics. Ceram Int, 2018, 44: 8409-8416

49 Raccuglia P, Elbert K C, Adler P D F, et al. Machine-learning-assisted materials discovery using failed experiments. Nature, 2016, 533: $73-76$

50 Kingery D W, Bowen H K, Uhlmann D R. Introduction to Ceramics. 2nd ed. New York: John Wiley and Sons Inc, 1976

51 Nakayama M, Ohtake M. Exploration of perovskite-type dielectric materials by ab initio calculations and machine learning. Toyota Kenkyu Hokoku, 2016, 69: 141-142

\section{补充材料}

表 S1 BF-xBT 基固溶体陶瓷替代元素的特征及其性能总结表

本文以上补充材料见网络版 csb.scichina.com. 补充材料为作者提供的原始数据, 作者对其学术质量和内容负责. 


\title{
Effects of substitution element on physical properties of $\mathrm{BiFeO}_{3}-\mathrm{BaTiO}_{3}$ ferroelectric ceramics driven by data-mining oxide perovskites
}

\author{
Yinglong Jiang ${ }^{1,2}$, Jun $\mathrm{Li}^{1,2} \&$ Jian $\mathrm{Yu}^{2 *}$ \\ ${ }^{1}$ Department of Physics, Donghua University, Shanghai 201620, China; \\ ${ }^{2}$ Institute of Functional Materials, Donghua University, Shanghai 201620, China \\ * Corresponding author, E-mail: jyu@dhu.edu.cn
}

Data-mining from reliable extant data on basis of material informatics can lead to unveil those hidden qualitative and quantitative rules and make predictions of new materials faster and cheaper, which would reduce human synthesis effort than traditional trial-and-error way. In contrast to traditional scenario of material research initiated by scientists' intuition and in a chain of synthesis-observation-analyses of mechanism, modern material research will change into productions and applications of data provoked by data science paradigm, while the present bottleneck of material informatics is still limited by the number of data. In the field of functional materials of ferroelectric piezoceramics, there are two ongoing trends of developing lead-free piezoceramics with thermal aging stability and high temperature piezoceramics with higher response for applications like in Internet-of-Things (IoT) and smart systems. Recent years, data-mining on oxide perovskites and designing new perovskite-type high temperature piezoceramics have been carried out and progresses were firstly reviewed in a short. Based on those quantitative relationships and rules data-mined out previously, various kinds of substitution elements for $\mathrm{BiFeO}_{3}-\mathrm{BaTiO}_{3}(\mathrm{BF}-x \mathrm{BT})$ solid solution ferroelectrics were proposed on basis of the mass and cation size features of atom and their effects on ferroelectric phase transition temperature, dielectric and piezoelectric properties observed experimentally. The compositions were tentatively chosen near the rhombohedral-pseudocubic structural phase boundary in order to check predominant contribution from substitution element. Among those elements of $\mathrm{Al}, \mathrm{Sc}, \mathrm{Zn}_{1 / 2} \mathrm{Ti}_{1 / 2}$ substitution for $\mathrm{Fe}$ and $\mathrm{Bi}_{1 / 2} \mathrm{Nb}_{1 / 2}, \mathrm{Al}_{1 / 2} \mathrm{Nb}_{1 / 2}, \mathrm{Zn}_{1 / 3} \mathrm{Nb}_{2 / 3}, \mathrm{Zr}$, Sn for Ti, it was demonstrated experimentally that $\mathrm{Bi}\left(\mathrm{Zn}_{1 / 2} \mathrm{Ti}_{1 / 2}\right) \mathrm{O}_{3}$ (BZT) exhibits a very promising effect in the BF- $x \mathrm{BT}$ system to obtain piezoceramics prepared using conventional solid state electroceramic processing with robust insulating and low dielectric loss properties fulfilled for the requirements of engineering applications. For those low dielectric loss BF- $x \mathrm{BT}-y \mathrm{BZT}$ ternary solid solution ceramics, their ferroelectric Curie temperature $\left(T_{\mathrm{C}}\right)$ follows a quadratic polynomial relation of $T_{\mathrm{C}}(\mu)=a+b \mu+c \mu^{2}$ and reduced mass of unit cell $(\mu)$ is a good descriptor to represent BF- $x$ BT and BF- $x$ BT- $y$ BZT solid solution perovskite numerically. Full poling for piezoelectricity was obtained in Mn-doped BF- $x$ BT- $y$ BZT $(x \leqslant 0.32, y<0.05)$ ceramics. So far, two ferroelectric piezoceramic materials were selected out: one is Mn-doped 0.74BF-0.22BT-0.04BZT exhibiting piezoelectric constant $d_{33} \sim 75 \mathrm{pC} / \mathrm{N}$, dielectric constant $\varepsilon_{33}{ }^{\mathrm{T}} / \varepsilon_{0} \sim 260$, dielectric loss tan $\delta \sim 0.01$, thickness electromechanical coupling factor $k_{t} \sim 0.42$, radial electromechanical coupling factor $k_{p} \sim 0.29$ and $T_{\mathrm{C}} \sim 630^{\circ} \mathrm{C}$, four times piezoresponse of commercial K-15 Aurivillius-structured bismuth titanate ceramics; another is Mn-doped 0.69BF-0.27BT-0.04BZT exhibiting $d_{33} \sim 145 \mathrm{pC} / \mathrm{N}, \varepsilon_{33}{ }^{\mathrm{T}} / \varepsilon_{0} \sim 570, \tan \delta \sim 0.03, k_{t} \sim 0.41, k_{p} \sim 0.33, T_{\mathrm{C}} \sim 510^{\circ} \mathrm{C}, 50 \%$ beyond K-81 tungsten-bronze-structured lead metaniobate ceramics. Similar to $\mathrm{PbZr}_{1-x} \mathrm{Ti}_{x} \mathrm{O}_{3}(\mathrm{PZT})$ system, BF-BT-BZT system exhibits a structural phase boundary and thus their piezoelectric performance and thermal stability could be modified in a wide range through adjusting composition and doping. At this point, it is time performing systematical characterizations on BF-BZT-BT perovskite-type piezoceramics for high temperature engineering application testing. Based on the correlation between $d_{33}$ and $\varepsilon_{33}$ for piezoceramics data-mined out previously, how to reduce synthesis effort for designing new materials was also argued using "bad" dielectric data of those high dielectric loss samples. Our essay demonstrates data-mining driven designing based on material informatics sure able to reduce time-to-insight and human effort on synthesis, accelerating new materials discovery and deployment.

perovskite-type oxides, data-mining, piezoceramics, ferroelectric phase transition, piezoresponse 